\title{
Somatic signals counteract reproductive aging in females
}

Ronald E Ellis ${ }^{1 *}$ and Qing Wei ${ }^{1,2}$

\begin{abstract}
Recent research shows that declining oocyte quality with age is not inevitable in nematodes, and similar signals might regulate reproductive aging in women.
\end{abstract}

Females are born with their complete complement of oocytes or egg cells. Although these oocytes can remain arrested for decades, during aging they decline in quality or undergo programmed cell deaths. Using the nematode Caenorhabditis elegans as a model, Luo et al. [1] showed recently that mutations in the insulin/insulin-like growth factor (IGF) and transforming growth factor (TGF)- $\beta$ signal transduction pathways improve oocyte quality in older females. Furthermore, genetic mosaic analyses revealed that each of these regulatory pathways acts in a different somatic tissue to control reproductive aging. Thus, declining oocyte quality is not inevitable in these worms, but seems to be determined by regulatory controls that balance somatic and reproductive needs. Given that the insulin/IGF and TGF- $\beta$ signaling pathways are conserved, these results raise the possibility that somatic signals regulate the quality of oocytes in older women.

\section{Conservation of declining reproductive success from worms to humans}

As a woman ages, her chance of conceiving a healthy child declines precipitously (reviewed in [2]). During this period, many of her oocytes are lost through programmed cell death, but reproductive problems predate their complete depletion. In particular, even oocytes that survive and mature are of lower quality than those found in young women. Defects in these oocytes lead to a decreasing chance of fertilization and an increasing risk of miscarriage or of a child with birth defects. Much of

\footnotetext{
*Correspondence: Ron.ellis@umdnj.edu

'Department of Molecular Biology, The University of Medicine and Dentistry of New Jersey, B303 Science Center, 2 Medical Center Drive, Stratford, NJ 08084, USA Full list of author information is available at the end of the article
}

this decline in oocyte quality is due to errors in handling chromosomes that lead to aneuploidy or triploidy (reviewed in [3]). Additional factors also contribute to lower oocyte quality but remain poorly understood.

Although nematodes have very short lifespans, several assays reveal that their oocytes also decline in quality with age. Perhaps the simplest method is to measure the probability that an oocyte will produce a healthy worm after fertilization. Using this definition, oocyte quality declines during normal aging in nematodes [4], as it does in women. In this new study, Luo et al. [1] extended these observations by showing that the frequency of nondisjunction in oocytes also increases with age in nematodes, as in women. Furthermore, the size of oocytes and their ability to be fertilized decline with age (Figure 1), and morphological defects increase in frequency.

\section{Improvement in oocyte quality by lowering TGF- $\beta$ or insulin/IGF signaling}

Genes that regulate oocyte quality are hard to identify by mutant screens. Because oocytes are among the most complex cells in existence, thousands of genetic or physical changes can decrease their ability to function. Thus, Luo et al. [1] tested regulatory genes that were known to influence the animal's development or lifespan to see whether they also affected reproductive aging. They found that changes in two different regulatory pathways improve oocytes in older females.

First, they tested a mutation that lowers the activity of the daf-2 gene, which encodes the $C$. elegans ortholog of the insulin/IGF receptor (reviewed in [5]). Lowering the activity of this signal transduction pathway increases the animals' lifespan by allowing the translocation of the DAF-16/FOXO transcription factor to nuclei, and also extends the reproductive span by several days [6]. Luo et al. [1] showed that decreasing daf-2 activity prevents the normal decline in oocyte quality with age, and that it also lowers the frequency of oocytes with chromosomal abnormalities.

Second, they [1] analyzed a mutation in sma-2, which encodes a SMAD protein that acts in the TGF- $\beta$ Sma/ Mab signal transduction pathway. Although these genes regulate body size and do not affect lifespan, decreasing 


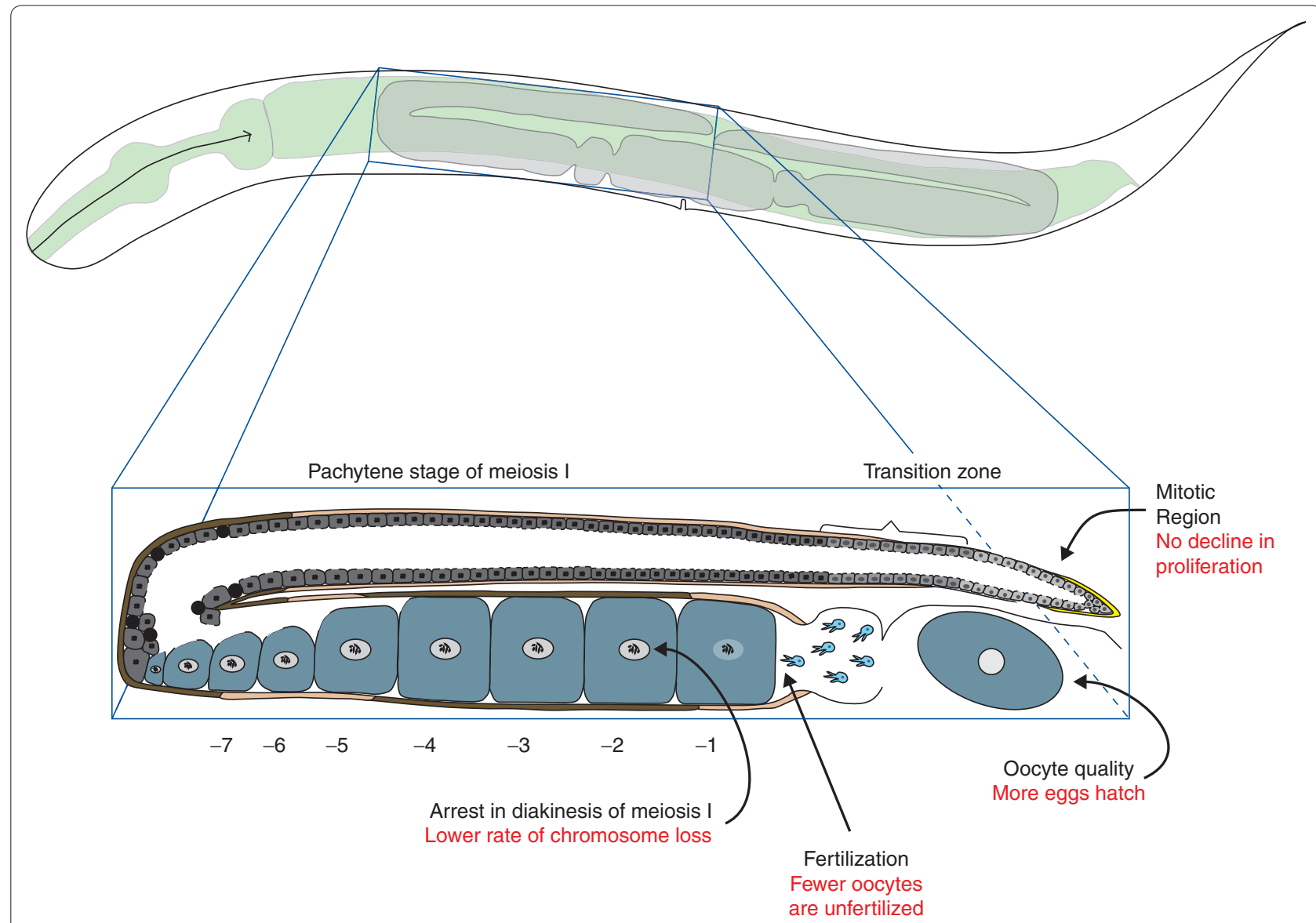

Figure 1. Mutations in the insulin/IGF or TGF- $\beta$ signaling pathways alleviate many symptoms of female reproductive aging. The outline of an adult hermaphrodite is shown at the top, with the gonad in gray. One arm of the gonad is shown in detail below. The distal tip cell (yellow) promotes mitosis. Developing oocytes are in gray, with the darkness of shading indicating their age. Sperm are shown in blue and are located in the spermatheca. Oocytes are numbered backwards from the proximal end; the -1 oocyte is undergoing maturation and the -2 to -7 oocytes are arrested in diakinesis (the last stage of prophase). Red text indicates the changes that are seen in insulin/IGF or TGF- $\beta$ pathway mutants.

their activity can extend the reproductive span of these nematodes [7], improve oocyte quality and decrease the frequency of chromosome loss [1].

These mutations in the insulin/IGF and TGF- $\beta$ signal transduction pathways have broad effects on reproduction (Figure 1) because they also promote the proliferation of germline stem cells in older animals $[1,8]$ and help alleviate the effects of $\gamma$-irradiation on oocyte quality [1].

\section{The insulin/IGF and TGF- $\beta$ signaling pathways act in the soma to control oocyte quality}

To determine where these genes act to regulate oocyte quality, Luo et al. [1] used a variety of techniques to create genetic mosaic animals and measured their reproductive spans and studied the morphologies of developing oocytes.

The DAF-2 receptor acts by repressing the DAF-16/ FOXO transcription factor. Thus, mutations in daf-16 block the extension of the reproductive span caused by lowering daf-2 activity. However, Luo et al. [1] show that the expression of DAF-16 in either the muscle or the intestine allows the extension of the reproductive span (Figure 2). These results imply that insulin/IGF signals work in these somatic tissues to control the somatic expression of DAF-16. However, what target genes DAF-16 regulates, and how these target genes influence the quality of oocytes in the germline, remain unknown. Moreover, independent studies of germ cell proliferation indicate that DAF-16 functions in the germline to regulate this trait [8]. Thus, the insulin/IGF pathway might act in both the soma and germline to control oogenesis. How its effects on germ cell proliferation, reproductive span and oocyte quality are related is unclear.

Similar studies suggest that the TGF- $\beta$ pathway acts in the hypodermis to regulate oocyte quality (Figure 2). Furthermore, this effect can be separated from its influence on body size. To generate a list of genes that might be involved in reproductive aging, Luo et al. [1] used microarray analysis to compare transcripts found in 


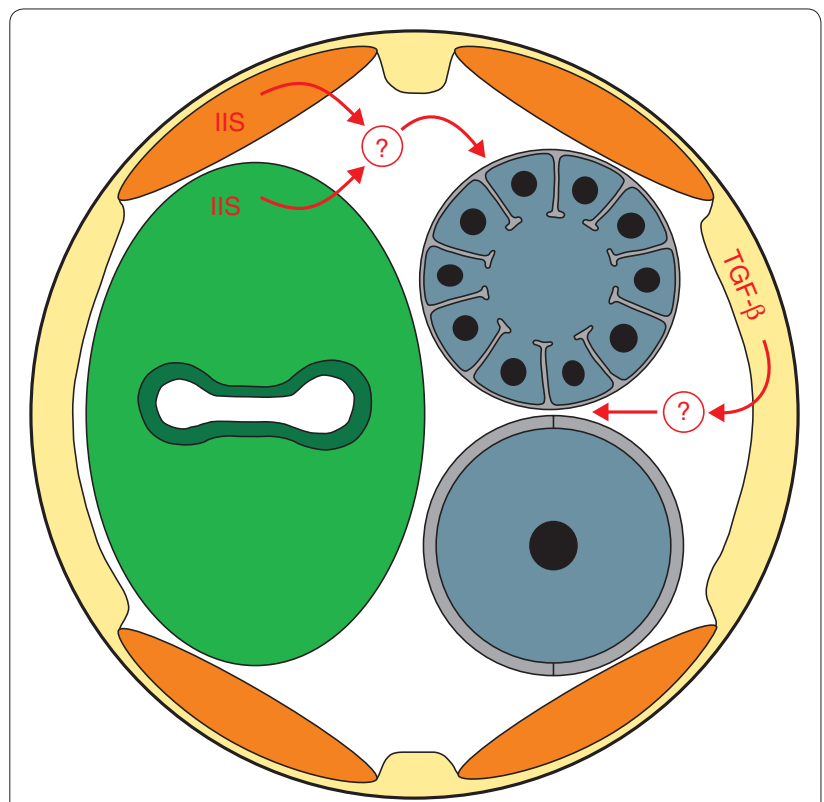

Figure 2. Somatic signals control female reproductive aging. A diagrammatic cross-section of an adult hermaphrodite is shown, with the hypodermis and nerve cords in yellow, the four muscle quadrants in orange and the intestine in green. The two sections of the gonad are gray, with the distal one containing numerous oocytes in pachytene and the proximal one containing a single large oocyte arrested in diakinesis. Insulin/IGF signals (IIS) and TGF- $\beta$ Sma/Mab signals are shown in red, with question marks to indicate that the means of communication with the germline remain unknown.

oocytes laid by old wild-type or sma-2 mutant animals. Although these unfertilized oocytes had probably undergone maturation and endomitosis (replication of chromosomes without cell division), it is possible that their transcript profile still resembled that of immature oocytes in the germline. Indeed, many of the genes identified have conserved roles in oogenesis [1]. Furthermore, decreasing the activity of some of the genes that were upregulated in sma-2 oocytes shortened the reproductive span. Given that these transcripts might encode important components of oocytes or early embryos, knocking them down would be expected to cause problems. Thus, a critical test for the future will be determining whether the upregulation of any of these genes can slow reproductive aging.

\section{Nematodes as a model for human female reproductive aging}

C. elegans was originally chosen as a model animal because it grows quickly, has many offspring and can selffertilize. These traits depend on the production of new oocytes in adults, which does not occur in humans. However, many critical aspects of oogenesis are similar in these two species [9] and might reflect a conserved program for oogenesis that can be dissected in worms.
In both nematodes and the human fetus, germline stem cells are maintained in a protected niche by the somatic gonad. Furthermore, when germ cells first enter meiosis, human and nematode oocytes each share cytoplasm with their neighbors. Later, many oocytes in each species undergo programmed cell death during prophase of meiosis I. Moreover, both human and nematode oocytes arrest near the end of prophase and wait for a signal to mature. In each species this signal activates the mitogen activated protein kinase pathway. Finally, oocytes in both species are fertilized before meiosis has been completed and then extrude polar bodies. These common features imply that nematodes could be an excellent model for many aspects of human reproductive biology.

In summary, recent findings show that conserved regulatory pathways control oocyte quality in aging nematodes, including the insulin/IGF and TGF- $\beta$ pathways described by Luo et al. [1], the DNA-damage response pathway (reviewed in [10]) and the core apoptotic pathway [4]. These findings might shed light on the molecular nature of reproductive aging in human females.

However, these studies also raise many new questions. To what extent are different aspects of reproductive aging in females related to each other? What regulatory changes occur in oocytes in response to the somatic signals described by Luo et al. [1]? How does the allocation of materials and nutrients to developing oocytes change during aging? What role do checkpoint processes have in maintaining oocyte quality in older worms? And most importantly, what processes cause the missegregation of chromosomes in older oocytes? Fortunately, the short lifespan of C. elegans will allow detailed studies of how oocyte quality changes during aging, which should lead to further breakthroughs.

\section{Acknowledgements}

This work was supported by NIH grant GM085282. We thank David Greenstein for comments.

\section{Author details}

'Department of Molecular Biology, The University of Medicine and Dentistry of New Jersey, B303 Science Center, 2 Medical Center Drive, Stratford, NJ 08084, USA. ${ }^{2}$ Graduate School of Biomedical Sciences, The University of Medicine and Dentistry of New Jersey, B303 Science Center, 2 Medical Center Drive, Stratford, NJ 08084, USA.

Published: 30 November 2010

\section{References}

1. Luo S, Kleemann GA, Ashraf JM, Shaw WM, Murphy CT: TGF-beta and insulin signaling regulate reproductive aging via oocyte and germline quality maintenance. Cell 2010, 143:299-312.

2. Broekmans FJ, Soules MR, Fauser BC: Ovarian aging: mechanisms and clinical consequences. Endocr Rev 2009, 30:465-493.

3. Hunt PA, Hassold TJ: Human female meiosis: what makes a good egg go bad? Trends Genet 2008, 24:86-93.

4. Andux S, Ellis RE: Apoptosis maintains oocyte quality in aging Caenorhabditis elegans females. PLoS Genet 2008, 4:e1000295.

5. Kenyon CJ: The genetics of ageing. Nature 2010, 464:504-512. 
6. Hughes SE, Evason K, Xiong C, Kornfeld K: Genetic and pharmacological factors that influence reproductive aging in nematodes. PLOS Genet 2007, 3:e25.

7. Luo S, Shaw WM, Ashraf J, Murphy CT: TGF-beta Sma/Mab signaling mutations uncouple reproductive aging from somatic aging. PLOS Genet 2009, 5:e1000789.

8. Michaelson D, Korta DZ, Capua Y, Hubbard EJ: Insulin signaling promotes germline proliferation in C. elegans. Development 2010, 137:671-680.

9. Govindan JA, Nadarajan S, Kim S, Starich TA, Greenstein D: Somatic CAMP signaling regulates MSP-dependent oocyte growth and meiotic maturation in C. elegans. Development 2009, 136:2211-2221.

10. Gartner A, Boag PR, Blackwell TK: Germline survival and apoptosis. In WormBook. Edited by The C. elegans Research Community. WormBook; 2008: doi/10.1895/wormbook.1.145.1.

doi:10.1186/gb-2010-11-11-142

Cite this article as: Ellis RE, Wei Q: Somatic signals counteract reproductive aging in females. Genome Biology 2010, 11:142 